\title{
Ziele notfallmedizinischer Behandlung
}

\author{
Franz-Josef Kretz, Jürgen Schäffer, Tom Terboven
}

F.-J. Kretz et al., Anästhesie, Intensivmedizin, Notfallmedizin, Schmerztherapie,

DOI 10.1007/978-3-662-44771-0_31, @ Springer-Verlag Berlin, Heidelberg 2016

Dieses Kapitel fasst kurz die wesentlichen Ziele der

notfallmedizinischen Behandlung zusammen.

Im Vordergrund der notfallmedizinischen Behandlung steht die Therapie von Störungen der Vitalfunktionen

- Atmung,

- Herz-Kreislauf-System,

- Wasser-Elektrolyt-Haushalt sowie Stoffwechsel und

- Gehirnfunktion.

Die Störung einer dieser Vitalfunktionen kann die Beeinträchtigung der anderen nach sich ziehen. Immer ist damit eine Gefährdung des Lebens verbunden. Ziel der Notfallmedizin ist es, die akute Lebensgefährdung von den Patienten abzuwenden. Die kausale Therapie basierend auf einer endgültigen Diagnose bleibt der Klinik vorbehalten. Die Ursachen der Störungen von Vitalfunktionen können sowohl im chirurgisch-traumatologischen als auch im internistischen Bereich liegen, das therapeutische Vorgehen ist jedoch im Wesentlichen gleich. Die Notfallmedizin ist damit ein interdisziplinäres Fach. 